\title{
SÍNDROMES EMPÍRICOS EN UNA POBLACIÓN ADOLESCENTE, EVALUADOS MEDIANTE EL YSR
}

\section{EMPIRIC SYNDROMES IN A YOUTH POPULATION, ASSESSED BY THE YSR}

\author{
Lilian R. Daset Carretto \\ Universidad Católica del Uruguay, Uruguay \\ Concepción López Soler \\ Universidad de Murcia, España \\ Hospital Universitario Virgen de la Arrixaca Murcia, España \\ María Dolores Hidalgo Montesinos \\ Universidad de Murcia, España
}

\begin{abstract}
Resumen: El estudio de poblaciones adolescentes desde un encare empírico y de corte epidemiológico, responde a la necesidad de contar con perfiles psicopatológicos y también de aspectos prosociales, que puedan ser útiles para la formulación de políticas en todos los ámbitos, máxime cuando los recursos de los países son escasos y las demandas en el sector van en aumento.

Este trabajo se centra en el estudio de las características psicopatológicas, sociodemográficas y competencias, en una población adolescente de una institución educativa de nivel secundario de la ciudad de Montevideo, Uruguay.

El objetivo específico desarrollado en esta comunicación, refiere al ajuste del Autoinforme para Jóvenes (Achenbach y colaboradores, 1978 y ss.) a población juvenil uruguaya; analizando la estructura factorial del mismo y contrastando estos hallazgos con los encontrados en otros países. Asi se comparan los hallazgos con los encontrados en estudios de López Soler en España (1998) y los del propio autor.

La estructura taxonómica obtenida contiene a los Síndromes de: Depresión-Ansiedad, Quejas Somáticas; Disocial; Oposición-Impulsividad; Alteración del Pensamiento; Retraimiento-Evitación, Inmadurez; Temor; Problemas Sociales y también al Síndrome Prosocial. Los resultados muestran coincidencias importantes con los trabajos de referencia.
\end{abstract}

Palabras clave: Adolescencia, Psicopatología, Taxonomías Empíricas, YSR.

\begin{abstract}
The study of adolescent populations, considered from an empirical and epidemiologic perspective, responds to the need for psychopathological profiles and for prosocial aspects, which may be useful in the policy formulation process in every field, especially when the country's resources are scarce and the sector's demands are increasing.

This work focuses on the study of the psychopathological and sociodemographic characteristics, as well as on the study of competencies, in an adolescent population of high school students in an educational institution of the city of Montevideo, Uruguay.

The specific objective developed on this paper, refers to the adjustment of Youth Self-Report (Achenbach et al, 1978) to a uruguayan youth population, analyzing the scale’s factorial structure and contrasting these findings with those found in other countries. Thus, our findings are compared with those originated by Lopez Soler in Spain (1998) as well as with those described by the scale's original author.

The taxonomic structure obtained comprises the following Syndromes: Depression-Anxiety, Somatic Complaints; Dissocial; Opposition-Impulsivity; Alteration in Thought Processes; Withdrawal-Avoidance, Immaturity; Fear; Social Problems and also, the Prosocial Syndrome. The results show significant coincidences with the studies of reference.
\end{abstract}

Keywords: Adolescence, Psychopathology, Empirical Taxonomies, YSR.

\section{INTRODUCCIÓN}

Alan E. Kazdin $(1993 ; 2003)$ planteaba -discurriendo sobre prevención y tratamiento en la Adolescencia- que la salud mental juvenil representa una área abandonada de investigación; "esto es -aún más evidente- en una región donde, los recursos disponibles para estudios psicológicos o de salud mental, son escasos o casi inexistentes" (Daset, 2005), así iniciábamos 
una de nuestras presentaciones hace ya unos años y hoy, hablar de problemas en la adolescencia o más aún, problemas de o con los adolescentes, parece ser una moneda corriente; pero no alcanzó igual preeminencia el estudio científico de esta etapa.

Los profesionales en Psicología -y de muchas otras áreas- nos enfrentamos a la necesidad de contar con una metodología de evaluación e intervención, que posibilite comprender y delinear estrategias y políticas para los grupos juveniles, sean éstos los protagonistas de las páginas rojas de los periódicos -en casi cualquier lugar del mundo- o los que forman parte de las huestes de jóvenes que, diariamente, se enfrentan a niveles de riesgo creciente ( $\mathrm{Ci}$ chetti, 2002). Todo esto dentro de un marco caracterizado por una multiplicidad de desafíos sociales y circunstanciales que, la globalidad, la aceleración de los cambios, las nuevas formas comunicacionales y las expectativas propias y de su entorno, amplifican. Tales necesidades hacen prioritaria la búsqueda de acercamientos metodológicos que den respuesta a las mismas.

La exigencia que la realidad impone al desarrollo científico-metodológico, se relaciona con la disponibilidad de un sistema de evaluación - de sustento empírico-, para el perfeccionamiento de estudios amplios y que, a su vez contenga la posibilidad de trabajo individual y con nuevos sujetos o momentos; especialmente cuando los resultados de múltiples investigaciones ponen en duda el trabajar con restricciones en la selección de poblaciones (Goodman, 1997 y 2001; Goodman et al, 2000). Sistemas de evaluación que estén en condiciones de ser replicados y usados en exploraciones longitudinales. Razones que - para un país en vías de desarrollo - cobran singular valor, al implicar un ajuste a realidad y un aporte al mejoramiento de la calidad de vida de sus jóvenes, a partir de información verificable y útil para el desarrollo de políticas educativas, sanitarias y sociales y la asignación de recursos; lo que señalaba Kessler como una de las virtudes de este sistema (2000).

Diferentes Modelos de Clasificación en Salud Mental se fueron desarrollando a lo largo de la historia.
En el siglo pasado fueron cobrando fuerza dos sistemas, el de la OMS en su décima edición (CIE-10, 1992) y el DSM-IV en su edición revisada de APA (2002); ambos se actualizan periódicamente, cada vez más a partir de datos de la investigación. Otros sistemas obedecen a una especificidad mayor, especialmente se empiezan a delinear los que buscan un estudio de mayor precisión en la infancia y adolescencia. EI GAP - por sus siglas en inglés Group for the Advancement of Psychiatry- ( $1^{\mathrm{a}}$. Edición en 1966; en Jiménez, 1997) ha tenido una proyección escasa, lo que muchos argumentan es que se debe al carácter difuso de sus definiciones diagnósticas (González Barron, 1998).

Dentro de los abordajes menos conocidos en Latinoamérica se encuentra el interesante trabajo de Masud Hoghughi (The Problem Profile Approach, 1992) desarrollado al final de los años 60 y revisado a principios de los 90; el mismo se sustenta en el concepto de "Problema" como condición, problema que se inscribe en Áreas, que son a las que debería apuntar el diagnóstico; para el análisis de datos utiliza medidas de tendencia central.

Destacan especialmente los trabajos de Rutter -a los que anteceden los de Greespan y Lurie (1981)-, que en las últimas décadas se han incrementado y se han extendido a otras etapas de la vida; cobrando adeptos en numerosos ámbitos de la Psiquiatría y Psicología (Sroufe \& Rutter, 1984; Rutter, 1989, 1994 y 2003; Rutter et al, 2000; Garrido Landivar, 1998; Cicchetti, Dante \& Rogosch, Fred, 2002; Rutter, 2003).

En este orden de cosas, buscando responder a asuntos de orden más específico en poblaciones particulares y a la vez, encontrar posibles comunalidades en los grupos etáreos y compararlos según variables sociodemográficas y prosociales, se plantea el Sistema de Evaluación Basado Empíricamente (ASEBA), tras años de investigación de Thomas $M$. Achenbach y su grupo de colaboradores (Achenbach et al, 1978 y s.s.), el que actualmente cuenta con múltiples estudios en todo el mundo y ha sido uno de los referentes en el desarrollo de éste tipo de metodología plasmada en variadas adaptaciones. 
A las anteriores se suman otras aproximaciones diagnósticas que también contienen -en mayor o menor medida- criterios de clasificación que, como plantean Lahey y colaboradores (2004), transcurren en forma implícita -además de los "oficiales" DSM y CIE- y están representados sustantivamente por Achenbach, pero también se inscriben en éstos los trabajos de Conners y colaboradores $(1998$, ) con la "Conners Parent Rating Scale" (CPRS-R) y el Autoinforme desarrollado por Quay y Peterson en los años 80 (Revised Behavior Problem Checklist) (en Quay, 1993).

En coincidencia con lo planteado por Millon (2004), gran número de síndromes psicopatológicos son inespecíficos, con respecto a las causas; los síntomas y síndromes son, muchas veces, pautas reactivas que se ponen en marcha por las causas más diversas, sean estas causas primariamente psíquicas o somáticas. Considerados entonces bajo esta óptica, la búsqueda de información sobre qué síntomas y signos son los que se disparan con cierta uniformidad, se vuelve una tarea crucial y perentoria.

Para una mejor comprensión de los resultados, primeramente se expondrán -en el siguiente apartado- algunas de las características de la Evaluación basada Empíricamente.

\section{EVALUACIÓN Y CLASIFICACIÓN DESDE LA APROXIMACIÓN EMPÍRICA DE LAS TAXONOMÍAS}

Han surgido, en este escenario complejo y tras la necesidad de resolver los problemas que deja sin responder la aproximación ideográfica, acercamientos de tipo nomotético; que según T. Millon (2004) permitirán contar con modelos flexibles, pertinentes e integradores, para el diagnóstico, clasificación, terapéutica e investigación. Entre estos se cuentan aquellos que, tienen entre sus procedimientos principales, el análisis estadístico y a partir de él, el estudio cualitativo de los datos.

Para Heubeck, recordando los planteamientos de Feinstein (1967) y Mezzich y Mezzich (1987) - en Heubeck, 2000-, es relevante el Sistema de Clasificación al que adhiere el profesional, porque el mismo determino el modelo que luego usará para "observar, pensar, recordar y actuar".

Para desarrollar estudios que den como producto Taxonomías Empíricas (agrupaciones taxónicas estadísticamente claras que se obtienen de datos de poblaciones) debería atenderse -sin lugar a dudas- a lo planteado por Lenzenweger (2004): "solo debe emprenderse si se cuenta con un modelo científicamente legítimo; si el modelo sustenta la existencia de un taxón en forma clara y si la obtención de ese nuevo taxón supone una diferencia en la compresión de la estructura, proceso o modelo que esté a consideración, esto es en definitiva, si hace aportes novedosos a la ciencia".

Los resultados de las Taxonomías deben interpretarse como cualquier otra estadística (Meehl, 1999; 2001) y solo se puede hablar de problemas a nivel de estructura o forma, la identidad o cualidad de la información subyacente está en relación con el marco conceptual al que adscribe el científico, cuando infiere la relación o calidad de esos datos (Cronbach, 1972; Meehl, 1999).

La Taxonomía supone clasificar, pero además, conlleva el ejercicio de encontrar los taxones o grupos que darán lugar al modelo. Visto así, el sistema de Taxonomías de base empírica, comporta un proceso minucioso con unos objetivos predeterminados. La forma en que se realiza esa búsqueda, la verificabilidad y la reformulación, están íntimamente ligadas con el procedimiento usado para obtener las características que definen a los taxones; el matiz diferencial de este sistema obedece entonces al proceso empírico de recogida y análisis de la información sobre similitudes y diferencias.

La más difundida y desarrollada es la de Thomas M. Achenbach, a partir de sus trabajos pioneros con el Child Behavior Checklist, esto ocurría a fines de los 60 y principio de los 70 . Han habido y hay otras propuestas de aproximaciones taxonómicas, tal el caso de Quay y Peterson en los años 80 (Revised Behavior Problem Checklist) (Quay et al, 1987); Verhultz, y Howell, 1989 ( en Verhultz et al, 1997); Conners al (1998), con la "Conners Parent Rating Scale" (CPRS-R) y Goodman con 
un formato similar al CBCL, pero de menor extension (1997).

Entre sus antecesores, se cuenta a Ackerson, 1942; Hewit y Jenkins, 1946; Jenkins y Glickman, 1946, quienes comenzaron calculando correlaciones entre pares de ítems, buscando distinguir síndromes mediante la colaboración clínica y criterios estadísticos (en Achenbach, 1966; 1978; 1982; 1989; 1993).

La Taxonomía Basada Empíricamente está conformada por los Síndromes Empíricos -fenómenos que tienden a co-ocurrir-. La asignación de un individuo a un Síndrome es una cuestión de grado, pudiendo además tener puntuaciones en otros síndromes, esto es lo que la hace tan flexible, además de la gradación. En todos los casos, como plantea Achenbach (1991a), cualquier taxonomía debe verse como un medio provisorio para organizar el conocimiento, no obstante los beneficios que la misma pueda aportar, es sólo una piedra en el camino de la comprensión, sujeta a constante revisión y que no debería -en el estado actual de la cuestión- convertir los resultados en categorías incuestionables y cerradas, nada más alejado a su sentido original (Lambert et al,2007).

En los años sesenta, Thomas M. Achenbach ya desarrollaba el concepto de Taxonomía Empírica y proponía gran parte de lo que luego sería su Sistema de Evaluación Basado Empíricamente, ASEBA -Achenbach System of Empirically Based Assessment-; con la finalidad de contar con un diseño que facilite la valoración, planificación, intervención y evaluación de los resultados, en campos como la salud y educación, entre otros (Achenbach et al, 1987). La propuesta se sustenta en una serie de técnicas de evaluación empírica que luego dan lugar a la clasificación. Siguiendo así el camino inverso, que más habitualmente recorren los sistemas de mayor uso; lo que el mismo Achenbach Ilama "from the ground up", en contraste con los sistemas tradicionales que suponen una aproximación "from the top down". La estrategia implica la evaluación propiamente dicha, el análisis estadístico y clínico de la información obtenida y a partir de allí, la construcción de la Taxonomía.

Los principios que sustentan este sis- tema se derivan mayoritariamente de las teorías psicométricas, en tanto la evaluación de comportamientos y emociones se realiza a partir de una gradación cuantitativa, para cada fenómeno en cuestión (Achenbach,1991b). Según señalan Achenbach y Mc.Counaghy (1997), la utilización de criterios psicométricos en la valoración posibilita :

a) minimizar el margen de error que se da en razón de las variaciones en los procedimientos de recogida de información como son las entrevistas abier-

tas o semi-estructuradas, (Nunnally \& Bernstein,1994);

b) evitar los errores ocasionados por las diferencias idiosincráticas;

c) contar con la posibilidad de comparar el caso concreto con otros en igual rango $y$

d) trabajar con variables relacionadas al desarrollo, los normativos grupos de referencia deben formarse según índice de desarrollo por Ej. edad.

El Modelo de Evaluación Multiaxial para adolescentes, incluye cinco ejes, según se describen en el esquema que figura en la Tabla 1:

\begin{tabular}{|c|c|c|c|c|}
\hline $\begin{array}{c}\text { EJE } 1 \\
\text { INFORMACIÓN } \\
\text { DE PADRES }\end{array}$ & $\begin{array}{c}\text { EJE } 2 \\
\text { INFORMACIÓN } \\
\text { DE } \\
\text { PROFESORES }\end{array}$ & $\begin{array}{c}\text { EJE } 3 \\
\text { EVALUACIÓN } \\
\text { COGNITIVA }\end{array}$ & $\begin{array}{c}\text { EJE } 4 \\
\text { VALORACIÓN } \\
\text { FÍSICA }\end{array}$ & $\begin{array}{c}\text { EJE } 5 \\
\text { EVALUACIÓN } \\
\text { DIRECTAY } \\
\text { AUTOEVALUACIÓN }\end{array}$ \\
\hline $\mathrm{CBCL}$ & TRF & WISC/WAIS-R & Talla/Peso & YSR \\
\hline $\begin{array}{l}\text { Historia del } \\
\text { Desarrollo }\end{array}$ & $\begin{array}{l}\text { Calificaciones } \\
\text { escolares }\end{array}$ & $\begin{array}{l}\text { Valoración } \\
\text { académica }\end{array}$ & $\begin{array}{l}\text { Valoración } \\
\text { médica }\end{array}$ & DOF \\
\hline $\begin{array}{l}\text { Entrevista con } \\
\text { padres }\end{array}$ & $\begin{array}{l}\text { Entrevista con } \\
\text { profesores }\end{array}$ & $\begin{array}{c}\text { Evaluación } \\
\text { perceptual, } \\
\text { motora, lingüística } \\
\text { y comunicacional }\end{array}$ & $\begin{array}{l}\text { Valoración } \\
\text { neurológica }\end{array}$ & $\begin{array}{l}\text { Entrevista Clínica } \\
\text { Cuestionarios de } \\
\text { Personalidad, de } \\
\text { Autoconcepto y } \\
\text { de Habilidades. }\end{array}$ \\
\hline
\end{tabular}

Tabla 1: Esquema de los Ejes de Evaluación Multiaxial para Adolescentes basado en el de T.M. Achenbach (1991). Contiene mención de técnicas a modo de ejemplo.

Achenbach inicia su trabajo de investigación, analizando las historias psiquiátricas de niños y niñas y en 1978, junto a Craig Edelbrock desarrollan el primero de los instrumentos del sistema, el Child Behavior Checklist para edades de entre 4-18 años (CBCL/1418), el que tendría repetidas revisiones y ajustes (Achenbach, y Edelbrock, 1987).

El segundo instrumento que editan es el Teacher Report Form (TRF, 1986) y en 1987, 
la primer versión del Youth Self Report (YSR). Conformando así la trilogía que, por años, será la piedra angular del sistema; trilogía que posibilita una aproximación multi-informante, lo que facilita la obtención de un más amplio espectro de información sobre las mismas áreas, complementario y diferencial. Se han mantenido con su estructura inicial, con algunos cambios tras las revisiones de 1991 y 2001 (Achenbach y Rescorla, 2001; Ivanova et al, 2007)).

Posteriormente se fueron ampliando los ámbitos de aplicación hasta llegar a completar una batería de instrumentos que recorre las etapas desde la lactancia hasta el adulto mayor, incluida una técnica de evaluación paralela de otros informantes calificados ( en Daset, 1999; 2002; 2005).

En la Tabla 2 se resumen los instrumentos principales del ASEBA.

Los resultados de múltiples estudios se resumen muy bien en la Estructura Factorial Común que se presenta en el 2001 (Achenbach, Dumenci y Rescorla): S. de Problemas Afectivos; S. de Ansiedad; S. de Problemas Somáticos o Quejas Somáticas; S. de Déficit de Atención e Hiperactividad; S. Oposicionista Desafiante y S. de Problemas de Conducta; todos ellos presentes en el CBCL/6-18, TRF y YSR. En el año 1991, Achenbach plantea una agrupación sindròmica -también común al CBCL/618, TRF y YSR que se estructura con el S. deAnsiedad-Depresión; S. de Retraimiento;
S. de Quejas Somáticas; S. de Conducta Delictiva; S. de Conducta Agresiva; S. de Problemas Sociales; S. de Alteraciones del Pensamiento y $\mathrm{S}$. de Problemas de Atención.

Para ambas estructuras centroides se excluyen los ítems que refieren a aspectos Prosociales o de Deseabilidad Social y solo se trabaja con los datos de los reactivos que buscan encuestar aspectos psicopatológicos o desadaptados.

En tanto los trabajos del equipo de Murcia se fueron incrementando y también enriqueciendo con otras pruebas de cribado, especialmente en lo que refiere a Personalidad y a comportamientos específicos -delictivos, adicciones, etc.- (López Soler, López Pina y García Montalvo, 1998; López Soler, 1999; López Soler, Hidalgo Montesinos, Freixinos y López López, 1999; Freixinos Ros, 2000; López Soler y Pérez López, 2000; Martínez de Salazar, 2001; Lópz López y López Soler, 2008).

La estructura obtenida por el grupo de López Soler y colaboradores a partir del SYSR (1998) se configura con: S. de DepresiónAnsiedad; S. de Agresividad; S. Infrasocializado no agresivo; S. de Conducta Delictiva; S. de Quejas Somáticas, S. de Búsqueda de Atención; S. de Problemas de Pensamiento; S. de Problemas en las Relaciones Sociales; S. de Hiperactividad y Déficit Atencional y S. de Consumo de Sustancia-Problemas de Identificación Sexual.

\begin{tabular}{|c|c|c|}
\hline $\begin{array}{l}\text { AREA O TIPO DE } \\
\text { EVALUACIÓN }\end{array}$ & INSTRUMENTO & $\begin{array}{c}\text { TIPO DE } \\
\text { INFORMANTE }\end{array}$ \\
\hline \multirow{2}{*}{ PREESCOLARES $1 \frac{1}{2}=5$ AÑOS } & Child Behavior Checklist (CBCL/11/r-5) & PADRES \\
\hline & Caregiver-Teacher Report Form (C-TRF) & CUIDADOR-MAESTRO \\
\hline \multirow{3}{*}{ EDAD ESCOLAR 6-18 AÑOS } & Child Behavior Checklist 6-18 (CBCL/6-18) & PADRES \\
\hline & Teacher's Report Form (TRF/6-18) & DOCENTES \\
\hline & Youth Self-Report (YSR-11-18) & AUTOINFORME \\
\hline \multirow{2}{*}{ ADULTOS $18-19$ años } & Adult Behavior Checklist (ABCL/18-59) & PARES-CUIDADORES \\
\hline & Adult Self-Repot (ASR/18-59) & AUTOINFORME \\
\hline \multirow{2}{*}{ ADULTOS MAYORES $60-90+$} & Older Adult Behavior Checklist (OABC/60-90+) & CUIDADOR-PARES \\
\hline & Older Adult Self-Report (OASR/60-90+) & AUTOINFORME \\
\hline \multirow{3}{*}{$\begin{array}{l}\text { OBSERVACIÓN Y } \\
\text { ENTREVISTA }\end{array}$} & $\begin{array}{l}\text { Entrevista semiestructurada/Niños y } \\
\text { Adolescentes (SCICA) }\end{array}$ & PROFESIONAL \\
\hline & Test de Observación (TOF) & PROFESIONAL \\
\hline & Guía de Observación Directa (DOF) & $\begin{array}{l}\text { PROFESIONALY } \\
\text { OTROS }\end{array}$ \\
\hline
\end{tabular}

Tabla 2: Resumen de los instrumentos principales del Aseba (Daset, 2005) 
Los Síndromes descritos por Achenbach y colaboradores son comparados con las categorías diagnósticas DSM-IV y CIE-10, como se expresa en la Tabla Resumen (Martínez Salazar, 2001).En este orden de cosas y atendiendo a la compartida idea de necesidad que se delinea desde tan diversos ámbitos y autores, el propósito de este trabajo es el de presentar un acercamiento al tema de la evaluación -con un sistema de clasificación de sustento- a partir de los resultados de un estudio realizado en Uruguay con uno de los instrumentos del ASEBA en la versión de Lemos Fidalgo, Calvo y Menéndez (1992) en un grupo de adolescentes. Específicamente, el objetivo es conocer la estructura factorial del YSR en una población de adolescentes uruguayos y elaborar una Taxonomía de base empírica que se exprese en Síndromes, a partir del análisis del YSR según sexo y grupos de edad, así como analizar las estructuras específicas con el fin de determinan si existen síndromes comunes.

- Síndrome de Introversión /Internalizante y Trastorno Esquizoide de la Personalidad

- Síndrome de Quejas Somáticas y el Trastorno de Somatizac ión

- Síndrome de Depresión -Ansiedad y el Trastorno Depresivo Mayor; Trastorno por Ansiedad generalizada y Trastorno mixto ansioso-depresivo

- Síndrome de Problemas Sociales y el Trastorno de Personalidad por Dependencia

- Síndrome de Problemas de Pensamient o y el Trastorno por Déficit de Atención e Hiperactividad y los Trastornos Hipercinéticos

- Síndrome de Conducta Delincuente y Trastorno Disocial y Oposicionista Desafiante

- Síndrome de Agresividad o de Conducta Agresiva y Trastorno Negativista

Tabla Resumen: Adaptado de Martínez de Salazar, 2001.

\section{METODOLOGÍA}

\section{Sujetos}

La población del estudio estuvo constituida por 374 adolescentes de entre 12 y 19 años quienes completaron los instrumentos de evaluación-, El $41 \%$ pertenecen al sexo masculino y $59 \%$ al sexo femenino. La Media de Edad es de 14.7; en tanto para sexo femenino es de 14.7 y para sexo masculino es de 14.8.

La institución educativa, de nivel secundario, privado, fue seleccionada por conveniencia. Se enclava geográficamente en el área urbana, de la ciudad capital del país; el nivel socio-económico es medio y medio bájo según parámetro estándar y datos censales (ANEP, 2004).

\section{Instrumentos}

Autoinforme para Jóvenes (YSR, Achenbach y Edelbrock, 1987; Achenbach, 1991 a, 2001). en la adaptación española de Lemos, Fidalgo, Clavo y Menéndez (1992). La versión española fue adaptada lingüísticamente al español para Uruguay (Versión para UruguaySYSR-VU-, Daset, 1999). Los ajustes lingüísticos fueron menores y se realizaron luego de un estudio piloto con jóvenes de edades entre 12 y 18 años, ambos sexos. Un ejemplo de los ajustes realizados, en el ítems 7 de la $3^{a}$. Sección, es el siguiente: Versión española "Eres un poco chuleta"; en la Versión para Uruguay quedó como "Eres un poco engreído/a, compadre". Se usó también traducción inversa del inglés, revisando además las traducciones que se habían hecho al alemán y francés y las propias al español en uso en Estados Unidos. El instrumento consta de 3 secciones:

Sección 1: Datos generales, sociodemográficos y académicos; como edad, sexo, nivel educativo y actividad de cada uno de los progenitores, conformación del grupo familiar, así como, grado que cursa, orientación -si fuere del caso-, aplazamientos en primaria y secundaria, áreas académicas de preferencias y rendimiento autopercibido, etc.)

Sección 2: Aspectos pro-sociales, como por ejemplo vínculos con pares -cantidad y tipo de relación-, actividades de tiempo libre, deportes, pertenencia a grupos, actividades en el hogar, etc.

Sección 3: Tiene como objetivo encuestar 
comportamientos, pensamientos y emociones de los jóvenes, a partir de un listado de comportamientos desadaptados y otros ítems referidos a aspectos adaptativos; cubre un amplio espectro de los mismos.

La estructura general del Autoinforme supone una valoración por grados, siendo éstos entendidos como, la medida en que el fenómeno encuestado se presenta en la población. En la versión original, los grados están dados por una escala tipo Lickert, donde cada ítems puede ser respondido en alguna de los siguientes tres niveles: $0=$ No verdadero o Falso; $1=$ Algo o Algunas Veces y $2=$ Verdadero o La Mayoría de las veces. En la Versión para Uruguay se tomó como referencia la propuesta de Lopez Soler et al (1998) de una Lickert de 5 grados. Los ítems indagan sobre: "sentirse solo"; "sentir que no le quieren";" pensar que los demás le quieren perjudicar";"disfrutar de una buena broma o chiste"; "hacer valer sus derechos"; tener miedo a pensar o hacer algo malo", etc.

\section{Procedimiento}

El procedimiento de administración del Autoinforme es grupal -grupos de aproximadamente 20 jóvenes, de ambos sexos-, en el intervalo de una semana, previa gestión en la institución y obtención del consentimiento informado a padres. Se explicita a los jóvenes que la participación es voluntaria y la respuesta es con identidad reservada -sistema de doble sobre lacrado-, la identificación para todo el estudio es numérica.

Se realiza evaluación de la administración a través de diferentes criterios establecidos de antemano como, porcentaje de concurrencia sobre el total de la plantilla de alumnos; cantidad de cuestionarios respondidos sobre el total, así como, cantidad de ítems respondidos y nivel de ajuste de las respuestas.

Los cuestionarios fueron administrados por profesionales psicólogos y estudiantes avanzados, entrenados en las normas de administración de esta prueba y en trabajo con grupos. Un $95.4 \%$ del universo en estudio completó los cuestionarios; las deserciones obedecieron a inasistencias por enfermedad $-3.2 \%$ - y en los otros casos a la renuencia a participar.

\section{Análisis estadísticos}

Los procedimientos estadísticos utilizados para el análisis de los datos recogidos fueron: a) análisis descriptivos, con la finalidad de conocer la distribución en sexo y edad de la población;

b) reducción de datos y análisis de la dimensionalidad, a través del Análisis de Componentes Principales de 1er. Orden; con el mismo se obtuvieron los Síndromes de Banda Estrecha para los criterios de Eigenvalue $=1 \mathrm{y}$ selección de pesos factoriales $\geq .30$ (Comrey, 1988); con una Rotación Ortogonal de tipo Varimax;

c) análisis de la dimensionalidad en función de las variables sexo y edad;

d) estudio de la fiabilidad, a través del Coeficiente de Cronbach.

En el análisis de la dimensionalidad, una vez seleccionada la estructura general para 10 Factores, se indagó sobre: qué ítems cargaban en cada Factor, cuánto y el sentido de esa carga, de esta fase surgen los Síndromes Empíricos generales.

Posteriormente se desarrolló un estudio comparativo con los hallazgos de Achenbach et al (1991) y los de López Soler et al (1998). En segunda instancia se analizó cada ítems, en una lectura de tipo transversal, procurando conocer su comportamiento en el total del instrumento.

El análisis de la dimensionalidad según las variables sexo y edad, se orientó a la búsqueda de similitudes y diferencias, para lo que se realizaron sendos estudios factoriales, al igual que para los grupos de edad, menor de 15 años - de 12 a 14 años 11 meses 29 días- y con edad igual o mayor a 15 años - 15 a 19 años-.

Una tercera fase lo constituye la conformación de los Síndromes de Centro (Core Syndrome de Achenbach), los que se obtienen a partir de las estructuras por sexo, con aquellos Síndrome que tienen una coincidencia en más del $50 \%$ de sus ítems.

Las bases de datos utilizadas son: APA; Medline; EBSCO; Humanities y la propia base de trabajos de investigación conformada por el autor; además de publicaciones especializadas específicas.

La gestión de los datos se realiza por medio de la construcción de una base de datos.

El paquete estadístico de soporte para los análisis, es el provisto por el SYSTAT en su Sexta Versión (Wilkinson, 1993) y el SPSS (Norusis, 2000) en su versión $\mathrm{N}^{\circ} 10$. 


\section{RESULTADOS Y DISCUSIÓN}

Los procedimientos de análisis descriptivo según sexo y edad muestran la distribución que se muestra en el Gráfico 1. Con un mínimo de 12 años y un máximo de 19 , para un $41 \%$ de jóvenes de sexo masculino y $59 \%$ para femenino.

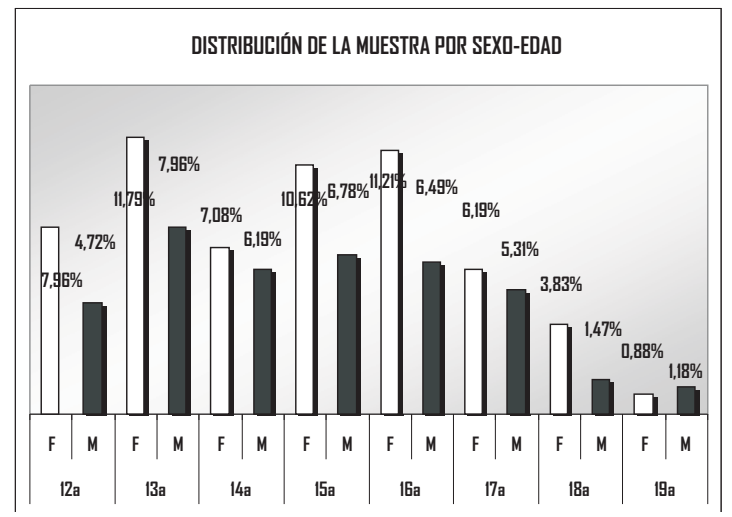

Gráfico 1: Distribución de la población según sexo y edad, con resultados porcentuales

\section{ESTRUCTURATAXONÓMICAYCORESYNDROMES}

Se realizaron diferentes Análisis de Componentes Principales exploratorios y se seleccionaron, para su estudio, las soluciones para 8,9 y 10 factores, por la similitud de conformación de muchos de sus Taxones -entre sí- y con otros estudios. Aún más, se aproximó el análisis a las estructuras de entre 8 y 12, todo lo que es coincidente con la Regla de Scree Plot (Hullin, Drasgow y Pearson, 1983; en López e Hidalgo, 1994).

Considerados los criterios estadísticos y clínicos, laelección recayósobrelaestructura de 10 factores, con una Varianza de 43.72, que explica un $35.83 \%$ del porcentaje total de la Varianza.

Esta estructura recoge la matriz general del estudio, con toda la población.

En la Tabla 3 se presentan los Síndromes de Centro o Core Syndromes.

\begin{tabular}{|c|c|c|}
\hline SiNDROME & VTE & $\%$ VTE \\
\hline Depresiòn -Ansiedad & 7.624 & 6.249 \\
\hline Prosocial & 5.072 & 4.16 \\
\hline Disocial & 5.04 & 4.131 \\
\hline Oposiciòn -Impulsividad & 5.967 & 4.891 \\
\hline Alteraciòn del Pensamiento & 3.579 & 2.934 \\
\hline Quejas Somàticas & 4.368 & 3.58 \\
\hline Retraimiento -Evitaciòn & 3.1 & 2.541 \\
\hline Problemas Sociales & 2.789 & 2.286 \\
\hline Temor & 2.77 & 2.27 \\
\hline Inmadurez & 3.403 & 2.79 \\
\hline
\end{tabular}

Tabla 3 Síndromes de Centro del estudio. (VTE=Varianza Total Explicada; \%VTE= Porcentaje de Varianza Total Explicada)
Las agrupaciones sindrómicas guardan un nivel alto de coincidencia con los hallazgos de Achenbach et al (1991b) y los de López Soler et al (1998), no obstante los factores extraídos fueron diferentes para el primer caso (8 en Achenbach, 1991b).

Los Síndromes extraídos son:

- Síndrome de Depresión-Ansiedad, con énfasis en los componentes de tipo depresivo, acompañado de algún elemento de alteración del pensamiento y de comportamiento de tipo confuso e impulsividad. Es altamente coincidente con los hallazgos de los trabajos de referencia y con otros de más reciente realización (Ferdinand, 2008; Najman et al, 2008; Kapi et al, 2007).

- Síndrome Prosocial, que reúne los ítems de la Escala de Deseabilidad Social de Achenbach et al (1991) y muchos de los que conforman el S. Infrasocializado no agresivo de López Soler et al (1998). Se caracteriza por tener dos agrupaciones diferenciales, la primera que contiene a los ítems con mayor peso factorial es netamente prosocial, en tanto en un segundo plano y con pesos entre .20 y .30 , se agrupan ítems del tipo que refiere al Llamado de Atención de López Soler et al (1998). En este Síndrome, surge la interrogante del peso que podría haber tenido el tipo de institución a la que asisten los jóvenes o si es parte de los valores compartidos por el universo en estudio. Al no contarse con estudios comparativos anteriores, se deja planteada la pregunta para los trabajos que se vienen desarrollando en el país; a la espera también de analizar el valor que los mismos puedan tener como Factores de Protección. - Síndrome Disocial, con el que se encuentran las mayores coincidencias con los hallazgos de López Soler et al (1998), en el Síndrome de Agresividad y con los de Comportamiento Agresivo y Comportamiento Delictivo de Achenbach et al (1998) y con el de Problemas de Conducta del mismo autor, en los Síndromes Integrados con DSM-IV (Achenbach et al, 2000; Vreugdenhil et al, 2006). Esta agrupación y en la misma línea argumental planteada en las limitaciones instrumentales, se vería más expuesta a sesgos culturales y aún por el peso de la subcultura adolescente, no obstante lo cual la coincidencia de los hallazgos es significativa y especialmente en lo concerniente a la heteroagresión y la antisocialidad como elementos comunes transculturalmente. Los ítems que refieren a la heteroagresión y los que 
netamente suponen un comportamiento delictivo podrían ser incluidos como Predictores de valor alto los primeros y medio los segundos; otros estudios también contienen similitudes (López López y López Soler, 2008).

- Síndrome de Oposición-Impulsividad, que además de contener elementos más típicos de la oposición-desafío, no llega a alcanzar el mismo tono del Trastorno planteado en el DSM-IV, caracterizándose más por control pobre de impulsos y una emocionalidad poco estable.

- Síndrome de Alteraciones del Pensamiento, que confirmaría los planteos de Achenbach (1991b) en cuanto a que, en todos sus estudios se estructura un factor de esta naturaleza, en lo que también coinciden Greembaum et al (1998), Verhulst et al (1997), López Soler et al (1998) y Ferdinand et al (2003). Se podría estar entonces en presencia de una conformación sindrómica que superaría los efectos de sesgo cultural y geográfico; reactivos más "puramente" psicológicos, esos que hicieron que las patologías del pensar fueran de las primeras en ser descriptas y tipificadas. A pesar de las coincidencias y de las posibilidades que este tipo de resultados ofrece, se está aún en terreno cenagoso, especialmente en edades tempranas y en los sujetos que puntúan en la franja de la M y la M+1DS. Todo esto sí puede y debe ser considerado para una detección más temprana o una intervención oportuna en dificultades con las que una orientación y apoyo primarios pueden colaborar.

Los ítems de este Síndrome deberían ser considerados como Predictores de valor medio, de posibles dificultades ulteriores, siguiendo el planteo de Ferdinand et al (2003; 2008).

- Síndrome de Quejas Somáticas, que constituye un "clásico" de las Taxonómicas empíricas, en los hallazgos del estudio se dan las mayores coincidencias con los estudios de referencia y otros con la misma metodología-en culturales occidentales y orientales-; constituye una especie de "factor común" al ASEBA más allá de las diferencias culturales y geográficas. Es en sí mismo un interesante hallazgo de las Taxonómicas, bastante coincidente con la preocupación por el cuerpo y lo somático que trae de su mano la globalización -entre tantos otros posibles argumentos-; sería deseable poder acceder a un estudio similar en poblaciones menos "culturizadas" e indagar si los tipos de quejas somáticas que se observan, son inherentes a la especie o es la cultura la que los direcciona.

- Síndrome de Retraimiento-Evitación, una estructura que coincide en forma parcial con los hallazgos de Achenbach et al (1991b) y López Soler et al (1998), encontrando su mayor correspondencia con los estudios de Weisz et al (2003) y de Ferdinand, van der Ende y Verhulst (2004). El posible valor predictivo de futuros episodios de autolisis -como señalara Wright, 1999- y el tipo de reactivos que lo componen, podría colocarlo entre las fortalezas del ASEBA, en cuanto a que este tipo de desórdenes parecería estar en la franja de los infradiagnósticados o de diagnóstico tardío.

- Síndrome de Problemas Sociales, con componentes que refieren sustancialmente a una falta de adaptación y en un segundo plano a labilidad emocional con cierta tendencia a la dependencia. Constituye una estructura difícil de "asir", pero que la clínica reconoce rápidamente bajo múltiples formas de demandas y acompañada de sintomatología perteneciente a otros grupos sindrómicos. Aún así, guarda cierta correspondencia con los hallazgos de los estudios de referencia.

- Síndrome de Temor, agrupación que contribuyó en la elección de la estructura de 10 factores, según criterio clínico. Su magra figura, destaca frente al sugerente llamador que constituye un Síndrome de Miedos y constituye en sí mismo una interrogante por su "trama argumental", no contando con hallazgos similares para poder hacer un estudio comparativo, como factor único. Si se encontraron algunas coincidencias con los estudios de Lahey et al (2004) sobre Ansiedad de Separación, Ansiedad Social y Miedos.

Este Síndrome es un producto diferencial de este estudio, que fue seleccionado por su valor clínico, no obstante persiste la duda de que la elección fuera la adecuada, aún tuviera una cierta presencia -casi constante- en otras estructuras factoriales generadas. Indudablemente una Escala de Miedos podría ser un buen complemento del SYSR-VU.

- Síndrome de Inmadurez, con una nominación que puede ser vista como paradójica para una población adolescente, pero que parece ser la más ajustada a la agrupación de ítems que constituyen este factor de 1er. orden. En los estudios comparativos encuentra cierta coincidencia con los hallazgos de Weisz et al (2003), en tanto se desdibujan las concordancias con 
los hallazgos de los autores de referencia de este estudio, entre múltiples síndromes. Este Síndrome, lleva a considerar la posibilidad de que se esté frente a un factor de diferente naturaleza que el resto, donde elementos de tipo desadaptativo podrían estar indicando jalones evolutivos, atendiendo más a la idea de adaptación que plantean Cichetti y Rogosch (2002). Entonces, una posible hipótesis a indagar es si cabría pensar en una agrupación que, más que un síndrome específico, conforme una estructura transversal a la propuesta taxonómica y permita discriminar en una escala ${ }^{1}$, niveles de maduración-adaptación, con la ventaja que ofrece el ASEBA de permitir incluir idiosincrasias culturales y de otros órdenes.

A las limitaciones mencionadas en cada Síndrome, se suman las que devienen de la elección de la estructura de 10 factores, razón por la que se realizan varios estudios paralelos, llegándose a trabajar con las estructuras por defecto hasta con agrupaciones de 2 y 3 factores como sugieren algunos autores -aún para los análisis de 1er. orden-. Los esfuerzos no arrojaron mejores respuestas, lo que no es razón para no seguirlas buscando.

\section{ESTRUCTURA TAXONÓMICA SEGÚN SEXO}

\section{ESTRUCTURAFACTORIALPARASEXOFEMENINO}

La estructura taxonómica para sexo femenino explica un $38 \%$ total de la Varianza. Su estructura es coincidente con muchos de los Síndromes de la General o de Core Syndrome.

El Síndrome de mayor peso es el de Depresión-Ansiedad como se muestra en la Tabla 4.

\section{ESTRUCTURA FACTORIAL PARA SEXO MASCULINO}

Las estructura taxonómica para sexo masculino explica un $42.5 \%$ de la Varianza y nuevamente se estructura con mayor fuerza el Síndrome de Depresión-Ansiedad, al igual que en los Core Syndrome y en la estructura para sexo femenino.

En la Tabla 5 se observa el mayor porcentual de varianza explicada del Síndrome de Búsqueda de Atención, frente al S. Prosocial que tenía el segundo peso más alto en chicas.

1Tal sistema siguió el propio Achenbach con la Escala de Deseabilidad Social.

\begin{tabular}{|c|c|c|c|c|}
\hline SÍNDROMES & MAX-MIN & M & DS & VTE \\
\hline Depresión-Ansiedad & $1-39$ & 15.6 & 7.4 & $8 \%$ \\
\hline Prosocial & $4-25$ & 20.4 & 3.2 & $4.4 \%$ \\
\hline Disocial & $0-21$ & 8.26 & 3.8 & $4.28 \%$ \\
\hline $\begin{array}{c}\text { Problemas de Atención y } \\
\text { Ansiedad }\end{array}$ & $1-32$ & 19.25 & 6.5 & $4.12 \%$ \\
\hline $\begin{array}{c}\text { Alteraciones de Pensamiento y } \\
\text { Temor }\end{array}$ & $1-20$ & 8.26 & 4 & $3.35 \%$ \\
\hline Quejas Somáticas & $0-17$ & 5 & 3.5 & $3.2 \%$ \\
\hline Búsqueda de Atención & $0-12$ & 6.9 & 2.25 & $3.0 \%$ \\
\hline Agresividad & $0-9$ & 4.4 & 1.78 & $2.81 \%$ \\
\hline Problemas Sociales & $0-16$ & 4.9 & 2.8 & $2.57 \%$ \\
\hline $\begin{array}{c}\text { Conducta Delictiva y } \\
\text { Oposicionista }\end{array}$ & $0-15$ & 5.6 & 2.8 & $2.45 \%$ \\
\hline
\end{tabular}

Tabla 4: Estructura Factorial para sexo femenino (Max-Min= Máximos y Mínimos; M= Media; DS= Desviación Estándar y VTE= Varianza Total Explicada porcentual)

\begin{tabular}{|c|c|c|cc|}
\hline SíNDROMES & MAX-MIN & M & DS & VTE \\
\hline Depresión-Ansiedad & $2-49$ & 16.7 & 9 & $\begin{array}{c}6.74 \\
\%\end{array}$ \\
\hline Prosocial & $4-22$ & 16.2 & 3.2 & $4.16 \%$ \\
\hline Búsqueda de Atención & $8-38$ & 22.9 & 6.5 & $5.27 \%$ \\
Inmadurez & $2-31$ & 14.3 & 6.6 & $4.68 \%$ \\
\hline Quejas Somáticas & $0-11$ & 3.8 & 2.4 & $3.38 \%$ \\
\hline Alteraciones del Pensamiento & $1-28$ & 11.4 & 4.8 & $4.29 \%$ \\
\hline Agresividad & $0-23$ & 5.8 & 3.7 & $3.88 \%$ \\
\hline Problemas Sociales-Evitación & $0-25$ & 8.1 & 3.9 & $3.59 \%$ \\
\hline Disocial & $1-21$ & 8.8 & 3.9 & $3.28 \%$ \\
Antisocial-Depresivo & $0-20$ & 3.78 & 3.08 & $3.09 \%$
\end{tabular}

Tabla 5: Estructura factorial para sexo masculino (Max-Min= Máximos y Mínimos; M= Media; DS= Desviación Estándar y VTE= Varianza Total Explicada porcentual)

\section{ESTRUCTURA TAXONÓMICA SEGÚN GRUPOS DE EDAD}

\section{ESTRUCTURAFACTORIALPARAEDAD<15AÑOS}

En esta estructura la Varianza explicada alcanza el 40\%; reiterando un mayor peso del Síndrome de Depresión-Ansiedad, pero en una conformación que también incluye la selección de aquellos ítems que refieren a Alteraciones del Pensamiento, por eso una nominación que incluye a ambos. 
En la Tabla 6 se observan la estructura taxonómica que se conformó para los jóvenes de entre 12 años y 14 años 11 meses y 29 días (Tabla 6).

\begin{tabular}{|c|c|c|c|c|}
\hline SÍNDROMES & MAX-MIN & M & DS & VTE \\
\hline $\begin{array}{c}\text { Depresión-Ansiedady } \\
\text { Alteraciones del Pensamiento }\end{array}$ & 3-53 & 18 & 9.9 & $7.64 \%$ \\
\hline Prosocial & $3-31$ & 22 & 4 & $5.07 \%$ \\
\hline Disocial & $2-32$ & 11 & 5.7 & $4.73 \%$ \\
\hline Quejas Somáticas -Depresión & $0-13$ & 3.18 & 2.6 & $3.13 \%$ \\
\hline Agresividad-Depresión & $0-28$ & 7.28 & 4.1 & $3.63 \%$ \\
\hline Somatización-Evitación & $0-24$ & 6.5 & 3.9 & $3.52 \%$ \\
\hline $\begin{array}{l}\text { Búsqueda de Atención- } \\
\text { Impuls ividad-Desafío }\end{array}$ & $1-28$ & 12.5 & 5.3 & $3.82 \%$ \\
\hline Temor & $0-6$ & 1.7 & 1.6 & $2.45 \%$ \\
\hline Oposición-Impulsividad & $0-16$ & 5.4 & 3 & $2.93 \%$ \\
\hline $\begin{array}{c}\text { Conducta Delictiva y } \\
\text { Evitación }\end{array}$ & $0-13$ & 2.8 & 1.9 & $2.87 \%$ \\
\hline
\end{tabular}

Tabla 6: Estructura factorial para jóvenes con edades menores de 15 años (Max-Min= Máximos y Mínimos; M= Media; DS= Desviación Estándar y VTE= Varianza Total Explicada porcentual)

\section{ESTRUCTURA FACTORIAL PARA EDAD $\Rightarrow 15$ AÑOS}

Esta estructura explica un $39.10 \%$ de la Varianza Total.

Nuevamente se configura un Síndrome de Depresión-Ansiedad que, a diferencia de lo que ocurre con las chicas, los reactivos que lo conforman son muy similares a los del estudio general y a los que están presentes en las taxonomìas para sexo femenino y masculino.

No obstante muestra interesantes particularidades, como por ejemplo el mayor peso de un Síndrome de Agresividad, lo que no se había dado en las otras estructuras (Tabla 7).

\begin{tabular}{|c|c|c|c|c|}
\hline SÍNDROMES & MAX-MIN & $\mathrm{x}$ & $\sigma$ & VTE \\
\hline Depresión-Ansiedad & $1-48$ & 19.9 & 8.4 & $8.13 \%$ \\
\hline Agresividad & $0-20$ & 6.8 & 3.6 & $3.94 \%$ \\
\hline Prosocial & $9-22$ & 18.5 & 2.4 & $3.88 \%$ \\
\hline Inmadurez-Depresión & $0-20$ & 10.3 & 4.2 & $3.52 \%$ \\
\hline Quejas Somáticas & $3-33$ & 15.5 & 5.4 & $4.26 \%$ \\
\hline Temor & $2-20$ & 9.8 & 3.3 & $3.25 \%$ \\
\hline $\begin{array}{l}\text { Alteraciones del Pensamiento- } \\
\text { Conducta Delictiva }\end{array}$ & $1-18$ & 5.1 & 3 & $3.22 \%$ \\
\hline Oposición-Impul sividad & $1-12$ & 6.7 & 2.26 & $3 \%$ \\
\hline $\begin{array}{c}\text { Hiperactividad-Déficit de } \\
\text { Atención }\end{array}$ & $1-14$ & 8.7 & 2.4 & $3.46 \%$ \\
\hline Inmadurez & $0-13$ & 6.9 & 2.3 & $2.42 \%$ \\
\hline
\end{tabular}

Tabla 7: Estructura factorial para jóvenes con edades iguales 0 mayores a 15 años (Max-Min= Máximos y Mínimos; M= Media; DS= Desviación Estándar y VTE= Varianza Total Explicada porcentual)

\section{ASPECTOS DIFERENCIALES POR SEXO Y GRUPOS DE EDAD}

Los análisis de las Estructuras Factoriales por Sexo y Edad muestran algunas constantes, que se resumen a partir de la agrupación de los Síndromes de Banda Estrecha, según el factor común de los elementos que definen al Síndrome (Gráfico 2).

Es así que, se obtiene un perfil común, donde se destaca un mayor número de problemas informados en el sexo femenino para los Síndromes de Depresión-Ansiedad, OposiciónImpulsividad, Retraimiento-Evitación y Quejas Somáticas, con lo que se coincide parcialmente con los hallazgos de Compas, Achenbach et al (1997). En tanto para Sexo Masculino se destaca el Síndrome Disocial.

La pregunta que surge -casi instantáneamente- es si se está frente a un tema de sexo o de género; si tendrán que ver las expectativas de rol para cada uno de los casos, sea para los problemas informados, como para las propias tendencias de respuesta sobre unos u otros asuntos.

Las agrupaciones por Edad muestran un incremento -en relación directa- para los Síndromes de Depresión-Ansiedad, Oposición-Impulsividad y Disocial. En tanto toma un sentido inverso -decrecen con la Edad- los Síndromes de Retraimiento-Evitación y de Alteraciones del Pensamiento; resultados similares obtuvieron Roth, Herzberg et al (2004).

El Síndrome Prosocial también acompaña inversamente la curva de crecimiento, a más edad menos Prosocialidad; lo que argumentarían muchos legos como la pérdida de la inocencia o es que los reactivos no son igualmente eficaces para edades mayores?, lo que se presume debería ser indagado primeramente para poder incluir o desestimar este resultado, hasta ese momento se lo toma como una tendencia.

La relación entre la variable Sexo y la Edad, para los Síndromes de Depresión-Ansiedad y Oposición-Impulsividad para chicas y el Síndrome Disocial en chicos, podría constituir un elemento de atención para las edades más bajas a medias y sería interesante ver su posible valor predictivo en futuros trabajos y avanzar en lo que refiere a factores de riesgo, lo que no deja de ser una tarea difícil en etapas de tantos cambios. 
No obstante la versatilidad, se refuerza la necesidad de las intervenciones tempranas y por ende de la evaluación precoz, aún a la luz de otros modelos de clasificación pueda impresionar como una "tenue" manifestación de Distress relacionados con la etapa evolutiva. este estudio; con el Objetivo General de obtener una estructura taxonómica explicativa de la psicopatología en una población adolescente, abordada con los instrumentos del sistema, posibilitando un estudio comparativo, con los hallazgos de Achenbach et al (1991) y López Soler et al (1998).

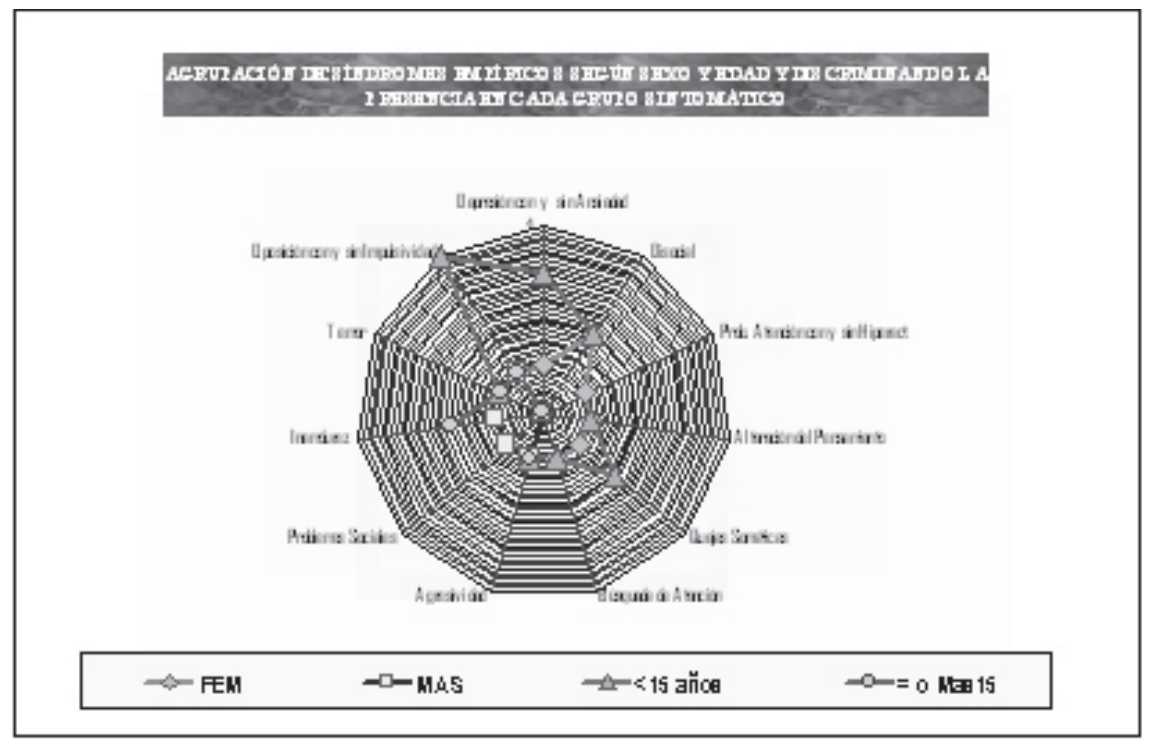

Gráfico 2: Agrupación sindrómica por edad y sexo según presencia de los grupos sintomáticos

\section{ANÁUSISDELARABIUDAD: CONSISTENCIAINTIERA}

La consistencia interna de la prueba es adecuada, según el Coeficiente Alpha de Cronbach cuyo valor es de .923, mostrando una buena fiabilidad de la escala en su conjunto y es similar y aún un poco superior al de los estudios de referencia, pero en todos los casos no ha sido inferior a .87.

\section{CONCLUSIONES}

Con un pie en el Modelo de Prototipos; otro en los desarrollos matemáticos aplicados a la estadística; bajo los soportes instrumentales de la informática y una tercer columna representada por la acumulación de hallazgos en el campo de la Evaluación en Psicología y más específicamente en Psicopatología (Quay et al, 1987; Achenbach \& McCounaghy, 1987; Verhulst et al, 1989; Jellinek \& Murphy, 1990) y una forma de construcción de la estructura taxonómica desde los cimientos (" el camino de la tierra al cielo"), es que Thomas M. Achenbach inicia el desarrollo del Sistema de Evaluación basado empíricamente (ASEBA), que aún hoy es una obra en construcción. Es en ese marco de la metodología de evaluación basada empíricamente que se ha desarrollado
A la luz de los objetivos propuestos y del análisis de los resultados obtenidos, se examinan algunos de los hallazgos más significativos:

La consistencia interna de la prueba es adecuada, según el Coeficiente Alpha de Cronbach cuyo valor es de .923, mostrando una buena fiabilidad de la escala en su conjunto.

Los procedimientos estadísticos dieron resultados dentro de lo esperado, en lo que refiere al comportamiento de las variables, pudiendo ser replicada la metodología utilizada por Achenbach et al (1991 y s.s.) y López Soler et al (1998 y s.s.) a través del Análisis de Componentes Principales y los estadísticos de tipo descriptivo.

Merece especial apartado la utilidad del Análisis de Componentes Principales en este tipo de estudios y las amplias posibilidades que brinda, entendido entonces, como uno de los insumos que forma parte de las fortalezas del Sistema de Evaluación basado empíricamente; la limitación tiene de suyo el tamaño muestral, por lo que también es una debilidad en este estudio para los grupos pequeños, razón por la cual se desestimó el estudio conjunto por grupo de edad y según sexo. 
En cuanto a limitaciones, a las ya mencionadas en cada Síndrome, se suman las que devienen de la elección de la estructura de 10 factores, razón por la que se realizan varios estudios paralelos, llegándose a trabajar con las estructuras por defecto hasta con agrupaciones de 2 y 3 factores como sugieren algunos autores -aún para los análisis de 1er. orden-. Los esfuerzos no arrojaron mejores respuestas, lo que no es razón para no seguirlas buscando.

Se plantean como retos de un futuro cercano, seguir indagando en el Síndrome de Depresión-Ansiedad, tanto por lo que de sí implica para una población juvenil, como por los aspectos de orden epidemiológico que aumentan las probabilidades riesgo para los grupos adolescentes. Este Síndrome y los problemas motivacionales y la labilidad emocional propia de esta etapa evolutiva, se constituyen en un factor de riesgo de entidad, además de las vulnerabilidades propias. Se aspira a poder considera este Síndrome en un estudio más amplio, donde se incluyan técnicas que evalúen adicciones, motivación, autocontrol y estilo de personalidad, buscando las posibles relaciones de todas ellas con variables sociodemográficas y de rendimiento académico.

Aunque con un peso factorial muy exiguo, pero conformando una entidad clínica de sumo interés, el Síndrome de Temor nos pone frente a un tema que, a nuestro entender ameritaría una profundización y estudios comparativos con otras culturas y en particular con jóvenes de medios urbanos y de medios rurales, con y sin enclave educativo institucional. Esto último supone un verdadero desafió para lo que habría que pensar en realizar estudios de tipo poblacional general, que permitan evaluar aqueIlas poblaciones juveniles que no están en el "sistema" sea este educativo, deportivo, etc., y que constituyen actualmente un problema para casi todas las sociedades urbanas.

Una consideración diferencial merece la $3^{a}$ Sección y los reactivos que indagan sobre aspectos prosociales, entre otros, donde la cultura -y aún la subcultura de la institución y el lugar residencial de pertenencia de los jóvenes- podría generar algún efecto de sesgo de tipo idiosincrático. A lo anterior se sumaría, el reconsiderar algunos reactivos que indagan sobre disocialidad o comportamientos delictivos, donde se pone de manifiesto el límite entre la norma y el uso, pudiendo también esto generar una tendencia de respuesta que confunda a la hora del análisis y nominación sindrómica. En esta sección las limitaciones son de una particular especie, en tanto pueden llevar al plano de discutir asuntos como los comportamientos delictivos de los jóvenes, ¿Se está frente a un Trastorno psicológico, moral, social?

Bajo el supuesto de que, los jóvenes de estos tiempos tienden a tener más aspectos comunes que diferenciales, -en razón de una globalidad que les atañe- aún las diversidades culturales, podría plantearse una cierta estructura psicopatológica básica común. El trabajo ha permitido atisbar esa estructura y también las diferencias que se pueden dar según el sexo, la edad - entre otros datos-. Variables todas que muestran - en diferentes gamasque detrás hay una relación, pero también que hay otras entidades que se deben seguir considerando, sean éstas el momento en que se produce la mayor convergencia, la intensidad de las mismas o esa condición individual, que hace que tomen un matiz único e irrepetible en cada persona.

\section{REFERENCIAS}

Achenbach, T. M. (1991b). Manual for the Child Behavior Checklist/4-18 and 1991 Profiles. Burlington: University of Vermont, Department of Psychiatry.

Achenbach, T. M. (1991c). Manual for the Teacher's Report Form and 1991 Profiles. Burlington: University of Vermont, Department of Psychiatry.

Achenbach, T. M. (1991d). Integrative Guide to the 1991 CBCL/4-18, YSR, and TRF Profiles. Burlington: University of Vermont, Department of Psychiatry.

Achenbach, T. M. (1997). Manual for Young Adult Self-Report and Young Adult Behavior Checklist. Burlington: University of Vermont, Department of Psychiatry.

Achenbach, T. M., \& McConaughy, S. H. (1997). Empirically based assessment of child and adolescent psychopathology (2nd ed., Vol. 13). Thousand Oaks, CA: Sage.

Achenbach, T. M., \& Rescorla, L. A. (2000). Manual for ASEBA Preschool Forms \& Profiles. Burlington, VT: University of Vermont, Research Center for Children, Youth, \& Families.

Achenbach, T. M., \& Rescorla, L. A. (2001). Manual for ASEBA School-Age Forms \& Profiles. Burlington, VT: University of Vermont, Research Center for Children, Youth, \& Families.

Achenbach, T. M., \& Rescorla, L. A. (2003). Manual for ASEBA Adult Forms \& Profiles. Burlington, VT: University of Vermont, Research Center for Children, Youth, \& Families. 
Achenbach, T. M., Conners, C. K., Quay, H. C., Verhulst, F. C. \& Howell, C. T. (1989). Replication of empirically derived syndromes as a basis for taxonomy of child/ adolescent psychopathology. Journal of Abnormal Child Psychology, 17, 299-323.

Achenbach, T. M., Howell, C. T., McConaughy, S. H., \& Stanger, C. (1998). Six-year predictors of problems in a national sample: IV. Young adult signs of disturbance. Journal of the American Academy of Child and Adolescent Psychiatry, 37, 718-727.

Achenbach, T. M., McConaughy, S. H., \& Howell, C. T. (1987). Child/adolescent behavioral and emotional problems: Implications of crossinformant correlations for situational specificity. Psychological Bulletin, 101, 213-232.

Achenbach, T. M., Newhouse, P.A., \& Rescorla, L. A. (2004). Manual for ASEBA Older Adult Forms \& Profiles. Burlington, VT: University of Vermont, Research Center for Children, Youth, \& Families.

Achenbach, T., Dumenci, L. \& Rescorla, L.A. (2002). Tenyear comparisons of problems and competencies for national samples of youth: self, parent, and teacher reports. Journal of Emotional and Behavioral Disorders, 4, 215-228.

Achenbach, T.M. \& Edelbock, C.S. (1987). Manual for the Youth Self Report and profile. Burlington, VT: University of Vermont.

Achenbach, T.M. (1991a) Manual for the Youth Self Report and 1991 profile. Burlington VT: University of Vermont.

Achenbach, T.M. y Edelbrock, C. (1978). The classification of child psychopathology: A review and analysis of empirical efforts. Psychological Bulletin, 85, 1275-1301.

Achenbach, T.M., Krukowski, R.A., Dumenci, L., \& Ivanova, M.Y. (2005). Assessment of adult psychopathology: Meta-analyses and implications of cross-informant correlations. Psychological Bulletin, 131, 361-382

Achenbach, Thomas M. \& Dumenci, Levent (2001). Advances in Empirically Based Assessment: Revised CrossInformant Syndromes and New DSM -Oriented Scales for the CBCL, YSR, and TRF: Comment on Lengua, Sadowksi, Friedrich, and Fisher. Journal of Consulting and Clinical Psychology: 69 (4),699-702

Achenbach, Thomas M. (1995). Empirically Based Assessment and Taxonomy: Applications to Clinical Research. Psychological Assessment: 7 (3), 261-274.

ANEP en Cifras. http://www.anep.edu.uy/ANEP/anep_cifras.htm(Consultada 02-10-04).

Bellock, A.; Sandín, B. y Ramos, F. (1995). Manual de Psicopatología. Vol. 1 y 2. Madrid: McGraw-Hill.

Cicchetti, Dante \& Rogosch, Fred A. (2002). A Developmental Psychopathology Perspective on Adolescence. Journal of Consulting and Clinical Psychology, Vol. 70, No. 1, 6-20
Compas, Bruce E et al (1997). Gender Differences in Depressive Symptoms in Adolescence: Comparison of $\mathrm{Na}$ tional Samples of Clinically Referred and Nonreferred Youths. Journal of Consulting and Clinical Psychology, 65, (4), 617-626.

Comrey, A.L. (1988). Factor analytic methods of scales developments in personality and clinical psychology. Journal of Clinical and Consulting Psychology, 56, 754-761.

Conners, C. K., Sitarenios, G., Parker, J. D. A., \& Epstein, J. N. (1998). The revised Conners' Parent Rating Scale (CPRS-R): Factor structure, reliability, and criterion validity. Journal of Abnormal Child Psychology, 26, 257-268.

Cronbach, Lee J. (1972). Fundamentos de la Exploración psicológica. Madrid: Biblioteca Nueva.

Daset, L. R. (1998). Aproximación a una taxonomía empírica: estudio en jóvenes de Uruguay. Comunicación presentada al /l Congreso de la Asociación española de Psicología Clínica y Psicopatología. Libro de Resúmenes: 83-84. España.

Daset, L. R. (1999). La depresión en la adolescencia: una propuesta de estudio, Comunicación presentada en 2das. Jornadas sobre Depresión de la Pastoral de la Salud de la Iglesia Católica de Uruguay. Uruguay.

Daset, L.R. (1999) . Los trastornos de conducta en adolescentes: una aproximación empírica. Comunicación presentada en: Jornadas sobre Trastornos de Conducta en la Infancia y la Adolescencia. Ministerio de Salud Pública. Uruguay.

Daset, L.R. (1999). Sintomatología depresiva en la adolescencia. Comunicación presentada en 2do. Encuentro de Centros del Programa de Vigilancia Epidemiológica en Depresión (OPS/OMS).Uruguay.

Daset, L.R. (2002). De las Cogniciones a la Depresión: el camino hacia la tristeza. Prisma, 18: 178-191.

Daset, L.R. (2002). Depresión en la Adolescencia: una aproximación desde la evaluación empírica. Prisma, 18: $25-32$.

Daset, L.R. (2005). Psicopatología en la Adolescencia: relación entre aspectos psicopatológicos, variables sociodemográficos y competencias en una población de jóvenes uruguayos. Tesis doctoral. Murcia. España.

Daset, L.R., et al (2002). Síntomas de Depresión en Adultos: avances de un estudio exploratorio. Prisma, 18: 33-43.

Ferdinand RF.(2008). Validity of the CBCL/YSR DSM-IV scales Anxiety Problems and Affective Problems. Journal of Anxiety Disorder 22(1):126-34.

Ferdinand, R. F., Hoogerheide, K. N., van der Ende, J., Heijmens Visser, J. H., Koot, H. M., Kasius, M. C., \& Verhulst, F. C. (2003). The role of the clinician: threeyear predictive value of parents', teachers', and clinicians' judgment of childhood psychopathology. Journal of Child Psychology and Psychiatry, 44, 1-10. 
Ferdinand, Robert F. et al (2004). Parent-Adolescent Disagreement Regarding Psychopathology in Adolescents from the General Population as a Risk Factor for Adverse Outcome. Journal of Abnormal Psychology. Vol. 113, No. 2, 198-206

Freixinos, María de los Angeles (2000). Consumo de drogas, personalidad y conductas patológicas en adolescentes. Tesis Doctoral: Universidad de Murcia. España.

Garrido Landivar, Emilio (1998). Psicopatología Evolutiva. España: Universidad Pública de Navarra.

González Barrón, Remedios (Coord.), (1998). Psicopatología del niño y del adolescente. Madrid: Pirámide.

Goodman R (1997), The Strengths and Difficulties Questionnaire: A Research Note. Journal of Child Psychology and Psychiatry 38:581-586.

Goodman, R. (2001). Psychometric properties of the Strengths and Difficulties Questionnaire. Journal of the American Academy of Child and Adolescent Psychiatry, 40, 1337-1345

Goodman, R. ; Renfrew, D \& Mullick, M (2000). Predicting type of psychiatric disorder from Strengths and Difficulties Questionnaire (SDQ) scores in child mental health clinics in London and Dhaka. European Child \& Adolescent Psychiatry, 9, 129-124.

Greenbaum, Paul E. (1998). Hierarchical Confirmatory Factor Analysis of the Child Behavior Checklist/4-18. Psychological Assessment, 10 (2),149-155.

Greenspan, S.I., \& Lourie, R.S. (1981). Developmental structuralist approach to classification of adoptive and pathologic personality organizations: Infancy and early childhood. American Journal of Psychiatry, 138, 725-735.

Heubeck, Bernd G; (2000). Cross-cultural generalizability of $\mathrm{CBCL}$ syndromes across three continents: From the USA and Holland to Australia. Journal of Abnormal Child Psychology; Vol. 28, 5: 439-450.

Hoghughi Masud (1992) Assessing Child and Adolescent Disorders London: Sage Pubs.

Ivanova, M. Y; Achenbach, T.M; Rescorla, L.A; Dumenci, L.; Almqvist, F.; Bilenberg, N.; Bird. H., Broberg, A.G; Dobrean, A.; Döpfner, M., Erol. N.; Forns.M.; Hannesdotti. H.; Kanbayashi, Y.; Lambert. M.C.; Leung, P.; Minaei, A.; Mulatu, M.S.; Novik, T.; Oh, K.J.; Roussos, A.; Sawyer, M.; Simsek, Z.; Steinhausen, H.C.; Weintraub, S.; Winkler Metzke, C.; Wolanczyk, T.; Zilber, N.; Zukauskiene, R. \& Verhulst, F.C. (2007). The generalizability of the Youth Self-Report syndrome structure in 23 societies. Journal of Consulting and Clinical Psychologhy. 75(5):729-38.

Jellinek, M. S. \& Murphy, J. M. (1990). The recognition of psychosocial disorders in pediatric office practice: The current status of the Pediatric Symptom Checklist. Journal of Developmental and Behavioral Pediatrics, 11, 273-278.

Jimenez,M. (Ed.),(1997).Psicopatología Infantil. Málaga: Aljibe
Kapi, A.; Veltsista, A.; Sovio, U.; Järvelin, M.R. \& Bakoula, C. (2007). Comparison of self-reported emotional and behavioural problems in adolescents from Greece and Finland. Acta Pediatrica, 96(8):1174-9.

Kazdin , A. y Buela, G. (1997). Conducta antisocial en la Infancia. Madrid: Pirámide.

Kazdin, A.E. (1988). Child Psychotherapy: Developing and identifying effective treatments. NY: Pergamon

Kazdin, A.E. (1993). Tratamientos conductuales y cognitivos de la conducta antisocial en niños: Avances de la Investigación. Psicología Conductual, Vol I (1), 111-144

Kazdin, Alan \& Weisz, John (Eds.). (2003). Evidence-Based Psychotherapies for Children and Adolescents. New York: Guilford Press 2003,

Kessler, Ronald C. (2000). Psychiatric epidemiology: Selected recent advances and future directions. Bulletin of the World Health Organization, 78, 4.

Lahey, Benjamin B. et al (2004). The Structure of Child and Adolescent Psychopathology: Generating New Hypotheses. Journal of Abnormal Psychology, Vol. 113, 3: 358-385.

Lambert, M.C.; Essau, C.A.; Schmitt, N. \& Samms-Vaughan, M.E. (2007). Dimensionality and psychometric invariance of the Youth Self-Report Form of the Child Behavior Checklist in cross-national settings. Assessment, 14(3):231-45.

Lemos, S; Fidalgo, A, M.; Calvo, P. y Menéndez, P. (1992). Salud mental de los adolescentes asturianos. Psicothema, 2: 21-48.

Lenzenweger, M. F. \& Clarkin J. F. , (2004). Major theories of personality disorder. NY: Guilford Press.

López López, J.R. y López Soler,C. (2008). Conducta antisocial y delictiva en la adolescencia. Universidad de Murcia: España.

López Soler, C. ( 1994).Temas de Psicología de la Personalidad. Barcelona: PPU.

López Soler, C. (1999). Aportaciones del modelo de taxonomías empíricas al diagnóstico psicopatológico en infancia y adolescencia. Comunicación presentada al // Congreso de la Asociación española de Psicología Clínica y Psicopatología. Libro de Resúmenes: 80-81. España.

López Soler, C. y García Sevilla, J. ( 1997). Déficits Atencionales . Murcia: Ojos Solares.

López Soler, C. y López Pina, J.A. (1998). La depresión en la infancia desde la perspectiva de las taxonomías empíricas. Revista de la Asociación española de Psicología Clínica y Psicopatología, 3 (2) ,95-102.

López Soler, C., García Montalvo, C., Pérez López, J., Brito, Alfredo, Tejerína, María y Fernández Ros, E... (1998). Psicopatología en la adolescencia: Taxonomías empíricas, rasgos de personalidad y estrés. (Proyecto HUM96/46). Murcia, Programa Séneca del Plan Regional de Investigación, Desarrollo Tecnológico y del Conocimiento. 
López Soler, C., Pérez López, J. Y García Montalvo, C. (1999). Autocontrol y psicopatología en adolescentes. Comunicación presentada al: /l Congreso de la Asociación española de Psicología Clínica y Psicopatología. Libro de Resúmenes: pp. 82-83. España.

López, J.A. e Hidalgo, M.D. (1994a). Análisis de componentes principales y análisis factorial. En M. Ato y J.J. López (Eds.).Fundamentos de Estadística con SYSTAT. Madrid: ra-ma.

López-lbor Aliño, Juan J. \& Valdés Miyar, Manuel (dir.) (2002). DSM-IV-TR. Manual diagnóstico y estadístico de los trastornos mentales. Texto revisado. Barcelona: Masson

Martínez de Salazar, Alma D. (2001). Síndromes Empíricos en Psicopatología de la Adolescencia: Factorización de la Escala Youth Self Report de Achenbach. Tesis Doctoral: Universidad de Murcia

Meehl, P. E.(1999). Clarifications about taxometric method. Journal of Applied and Preventive Psychology, 8, 165174.

Meehl, P. E.(2001). Comorbidity and taxometrics. Clinical Psychology: Science and Practice, 8, 507-519

Millon, Theodore (2004). Biomedical Explanations of Psychopathology, the DSM, and Essential Necessities for Advancement of Diagnostic Classification An Essay Review. Journal of Psychotherapy Integration March Vol. 14, 1: 106-119.

Ministerio de Salud Pública del Uruguay. (2004) Informe Estadístico año 2004. www.msp.gub.uy/ignoticias/12063. xls

Norusis, (2000). SPSS, Version 10.0. SPSS, Inc.

Nunnally, J. C., \& Bernstein, I. H. (1994). Psychometric theory (3rd Ed.). New York: McGraw-Hill.

OMS (1992). CIE-10: Trastornos Mentales y del Comportamiento: Descripciones clínicas y pautas para el diagnóstico. Madrid: Meditor.

Organización Panamericana de la Salud. (1998) La Salud en las Américas, Publicación Científica, 569, (1) 193-194.

Quay, H C et al (1987). Psychopathology of childhood: from description to validation. Annual review of psychology. 02/1987; 38:491-532

Quay, H. (1993). The psychobiology of undersocialized aggressive conduct disorder: A theoretical perspective. Development and Psychopathology, 5, 165-180.

Rosch, Eleanor et al. (1976). Basic objects in natural categories. Cognitive Psychology.8, (3) 382-439.

Roth, Marcus \& Herzberg, Philipp Yorck (2004). A Validation and Psychometric Examination of the Arnett Inventory of Sensation Seeking (AISS) in German Adolescents. European Journal of Psychological Assessment, Vol. 20, (3), 205-214.

Rutter, Michael (1989). Annotation: Child Psychiatry disorders in ICD-10. Journal of Child Psychology and Psychiatry, 30, 499-513.
Rutter, Michael (1994). Beyond Longitudinal Data Causes, Consequences, Changes, and Continuity. Journal of Consulting and Clinical Psychology, Vol. 62, No. 5, 928-940.

Rutter, Michael (2003). Commentary: Causal Processes Leading to Antisocial Behavior. Developmental Psychology, Vol. 39, No. 2, 372-378.

Rutter, Michael; Giller, Henri \& Hagell, Ann (2000). La conducta antisocial de los jóvenes. Madrid: Cambridge University Press.

Schmidt, Norman B.; Kotov, Roman \& Joiner, Thomas E. Jr. (2004). Taxometrics. Toward a new Diagnostic Scheme for Psychopathology. Washington, DC: American Psychological Association.

Seiffe-Krenke, I. (2003) Testing Theorie of Romantic development from adolescence to young adulthood: Evidence of a developmental sequence. International Journal of Behavioral Development, 27, (6) 519- 531.

Shafto, Patrick and Coley, John D. (2003). Development of Categorization and Reasoning in the Natural World Novices to Experts, Naive Similarity to Ecological Knowledge. Journal of Experimental Psychology: Learning, Memory, and Cognition. Vol. 29, 4:641-649.

Smetana, J.G, Campione-Barr, N. y Metzner, A. (2006) Adolescent Development in interpersonal and societal context. Annual Review Psychological, 57, 255-284.

Sroufe, L.A. \& Rutter, M. (1984). The domain of developmental psychopathology. Child Development, 55: 17-29.

Steinberg, L. (2005) Cognitive and affective development in adolescence. Trends in Cognitive Sciences, 9 (2), 6974.

Verhulst. F.C., van der Ende, J. y Rietbergen, A. (1997) .Ten years time trends of psychopathology in Dutch children and adolescents: no evidence for strong trends. Acta Psychiatry Scandinavians, 96, 1,7-13.

Vreugdenhil, C.; van den Brink, W.; Ferdinand, R.; Wouters, L. \& Doreleijers, T.. (2006). The ability of YSR scales to predict DSM/DISC-C psychiatric disorders among incarcerated male adolescents. European Child and Adolescent Psychiatry, 15 (2): 88-96.

Weisz, John R. et al (2003). Syndromal Structure of Psychopathology in Children of Thailand and the United States. Journal of Consulting and Clinical Psychology, Vol. 71, No. 2, 375-385.

Wilkinson, L. (1993). SYSTAT: The system for statistic 6.0. Systat Inc. Evanstone, IL.

Wright, Jack C; Zakriski, Audrey L. \& Drinkwater, Matthew (1999). Developmental Psychopathology and the Reciprocal Patterning of Behavior and Environment Distinctive Situational and Behavioral Signatures of Internalizing, Externalizing, and Mixed- Syndrome Children. Journal of Consulting and Clinical Psychology Vol. 67, (1): 95-107 